\title{
Piezoelectric Polymer Oxide Semiconductor Field Effect Transistor (POSFET) Devices for Touch Sensing
}

\author{
Ravinder S. Dahiya ${ }^{1}$, Giorgio Metta ${ }^{1,2}$, Maurizio Valle ${ }^{2}$ \\ ${ }^{1}$ Italian Institute of Technology, Genoa, Italy, 16163 \\ ${ }^{2}$ University of Genoa, Genoa, Italy, 16145
}

\begin{abstract}
This work presents the POSFET based touch sensing devices and their experimental evaluation. POSFET touch sensing devices presented here are primarily developed for the robotic applications. The design of these devices is inspired from human sense of touch. These devices are implemented by spin coating thin $(\sim 2.5 \mu \mathrm{m})$ piezoelectric polymer (PVDF-TrFE) film, directly on to the gate area of MOS transistor. The polymer film is processed in situ. The POSFET device represents an integral "sensotronic" unit comprising of transducer and the transistor and are thus capable of 'sensing and processing at same site'. The POSFET touch sensing devices are tested in a wide range (0.15-5N) of dynamic normal forces and at different frequencies.
\end{abstract}

Index Terms - POSFET, Touch Sensor, Robotics, PVDF-TrFE, Piezoelectric Polymers, Piezo-FET

\section{INTRODUCTION}

Touch sensing, needed for tasks like manipulation and exploration, is an important component of a robotic platform. Movement of robots from the structured environment of manufacturing plants to the daily life has also added new tasks like safe interaction in a human-populated environment. The way robots interact with the environment is an important issue, as real-world objects exhibit rich physical interaction behaviors which depend on how heavy and hard the object is when hold, how its surface feels when touched, how it deforms on contact and how it moves when pushed. Such interaction behaviors can be better understood by touching or physically interacting with them - as humans do.

On the basis of location of the sensors on the robot's body, the sense of touch in robotics is classified as Intrinsic and Extrinsic touch sensing. In Intrinsic touch sensing - analogous of kinesthetic sense in humans - the sensors are located within the mechanical structure of robot's body and hence they do not come in contact with real world objects. On the other hand, in Extrinsic touch sensing - analogous to Cutaneous or tactile sense in humans - the sensors are located on the surface of robot's body and they, therefore, come physically in contact with the objects under observation. This work is related with Extrinsic touch sensing, which deals with measurement of contact parameters like contact force, hardness etc. by touching them.

Over the years, touch sensing technology has improved and many force/pressure sensors, using different materials and transduction methods, viz: Resistive/Piezoresistive, Quantum Tunneling, Capacitive, Optical, Ultrasonic, Magnetic, Piezoelectric etc. have been developed [4]. Most of these sensors are generally big in size and thus unsuitable for body sites like robot's fingertips where large numbers of sensors with high density are needed. Miniaturized touch sensor, using MEMS approach, have also been developed [5, 6]. However, due to fragile nature, such sensors are not even able to cover the range of force experienced by humans (see, Table I) in a normal manipulative task. Thus usage of such sensors is limited to applications where very small contact forces are to be measured. Mechanically flexible touch sensors, using organic FETs, have also been developed for large area skin type of applications [7, 8]. However, best organics are known to have a mobility of about $1 \mathrm{~cm}^{2} / \mathrm{Vs}$ versus $85 \mathrm{~cm}^{2} / \mathrm{Vs}$ for silicon based MOS devices [9], which limits their usage to recording of slow varying contact forces only. Moreover, the organic FET based touch sensor are too big to achieve the spatial acuity similar to that of humans fingertips (see, Table I) - though they are good enough to match spatial acuity of less sensitive body parts (e.g. belly) of human body.

This work presents novel POSFET touch sensing devices and their experimental evaluation. The POSFET touch sensing devices are fabricated by spin coating thin $(\sim 2.5 \mu \mathrm{m})$ film of piezoelectric polymer, PVDF-TrFE (Polyvinylidene FluorideTrifluoroethylene), directly on to the gate area of MOS transistor. The POSFET device, thus, represents an integral "sensotronic" unit comprising of transducer and the transistor and capable of 'sensing and partially processing at same site'. Besides this, the marriage of sensing material and the electronics would improve signal to noise ratio and hence the force sensitivity. The POSFET touch sensors, presented here, are designed keeping in view the tactile sensing parameters, given in Table I, of human fingertips. This paper is organized as follows: The concept and design of POSFET touch sensing

TABLE I

Key Features For Cutaneous/Tactile Sense At FINGERTIPS OF HUMANS [1-3]

\begin{tabular}{|l|c|}
\hline Feature & Value \\
\hline Mechanoreceptor Density & 100 in $1.0 \times 1.5 \mathrm{~cm}^{2}$ area. \\
\hline $\begin{array}{l}\text { Range of Forces involved during normal } \\
\text { manipulative tasks }\end{array}$ & $0.15-0.9 \mathrm{~N}$ \\
\hline Detectable Frequency range of vibration & DC-700 Hz \\
\hline Spatial Acuity & $1 \mathrm{~mm}$ \\
\hline Receptor level processing of contact data & Yes \\
\hline
\end{tabular}

IEEE Catalog Number: CFP0926C ISBN: 978-1-4244-3832-7 Library of Congress: 2009900354 
device is presented in section II and the experimental evaluation of POSFET touch sensing devices is presented in Section III.

\section{CONCEPT AND DESIGN OF A POSFET TOUCH SENSING DEVICE}

\section{A. Concept of POSFET}

A piezoelectric film working in the sensing mode generates a charge/voltage on application of a mechanical force/stress. This charge/voltage is proportional to the applied force/stress $[10]$ and they are approximately related as:

$$
Q_{t}=d_{33} \times F
$$

Where, $Q_{t}$ is the charge developed on a touch sensor or taxel due to applied force, $F$ and $d_{33}$ is the piezoelectric constant. With piezoelectric polymer film on the gate area of MOS device, as shown in Fig. 1, such a charge/voltage modulates the charge/current in the induced channel of MOS device. Thus, POSFET touch sensing devices directly convert force into voltage, which can be amplified by POSFET itself and further processed by an electronic circuitry that may be present on the same chip. In this sense, POSFET touch sensing device can also be compared with the mechanoreceptors - the receptors that sense the contact parameters in the human skin and convert them into partially processed electrical signals [3].

Somewhat similar, but with extended gate, approach has been used for the development of ultrasonic sensors [11] and touch sensors [12]. The extended gates, epoxy-adhered with PVDF (Polyvinylidene Fluoride) films, are used in these works. The extended gate approach brings the sensor and conditioning electronics closer and hence the overall response is better than that of conventional approach - in which, the sensor and conditioning electronics are placed at a distance. But, the extended gates also introduce a large substrate capacitance, which in turn, significantly attenuates the charge/voltage available at gate terminals of MOS transistors and benefits offered by closely located sensor and electronics, are not fully exploited [13]. POSFET touch sensing devices, on other hand, are free from such problems.

\section{B. Design and Implementation}

The POSFET touch sensing device, implemented on silicon substrate is shown in Fig. 2. Detailed fabrication process of POSFET touch sensing devices is described elsewhere [14]; however, the key points are presented here. The MOS device is fabricated by using the n-MOS technological module of a non standard $4 \mu \mathrm{m} \mathrm{Al}$ gate p-well ISFET/CMOS technology [15]. In order to match the spatial acuity of receptors in human skin, POSFET touch sensing devices are designed to have size of $1 \mathrm{~mm} \times 1 \mathrm{~mm}$. In fact, better spatial acuity can be obtained by reducing the size of transistor. To obtain large transconductance, n-MOS devices are designed to have $\mathrm{W}=7500 \mu \mathrm{m}$ and $\mathrm{L}=12 \mu \mathrm{m}$. A $2.5 \mu \mathrm{m}$ thick PVDF-TrFE

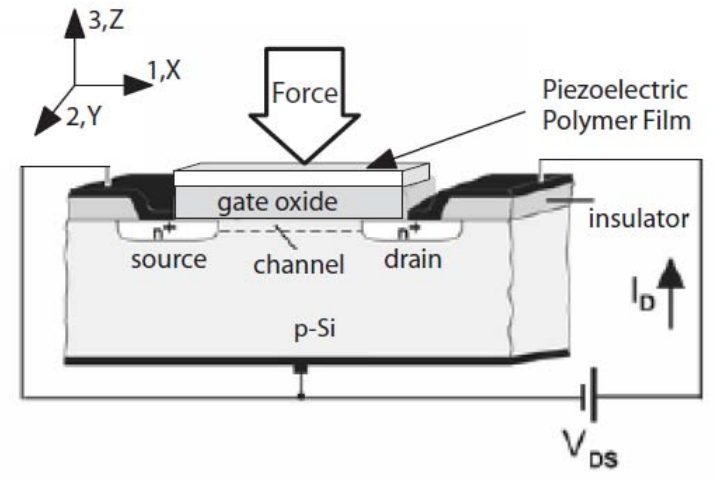

Fig. 1: The concept of POSFET touch sensing device.

piezoelectric polymer film is then spin coated from solution. A number of experiments, performed on dummy silicon wafers (i.e. without any MOS device), yielded that a $10 \%$ solution spin coated with $3000 \mathrm{rpm}$ for 30 seconds results in $\sim 2.5 \mu \mathrm{m}$ thick polymer film [16]. Further steps for in situ processing of polymer film include annealing at $120^{\circ} \mathrm{C}$ for three hours, followed by vacuum deposition of $200 \mathrm{~nm}$ metal $(\mathrm{Au} / \mathrm{Cr})$ on top of the polymer film and thereafter dry etching the polymer film. Piezoelectric polymers need to be polarized by applying a voltage at the rate of 80-100 V/ $\mu \mathrm{m}$ - which gets translated to 250 volts in present case. With such a high voltage, it is challenging to polarize the polymer film, in situ, without damaging or altering the characteristics of underlying MOS device. To overcome this challenging step, all metals layers and substrate under the polymer film were grounded before applying the voltage was across the polymer film. Thermal poling [17] was performed at $85^{\circ} \mathrm{C}$, to reduce the voltage needed for polarization - 200 volts was finally used. A further care was taken by applying the voltage in four cumulative
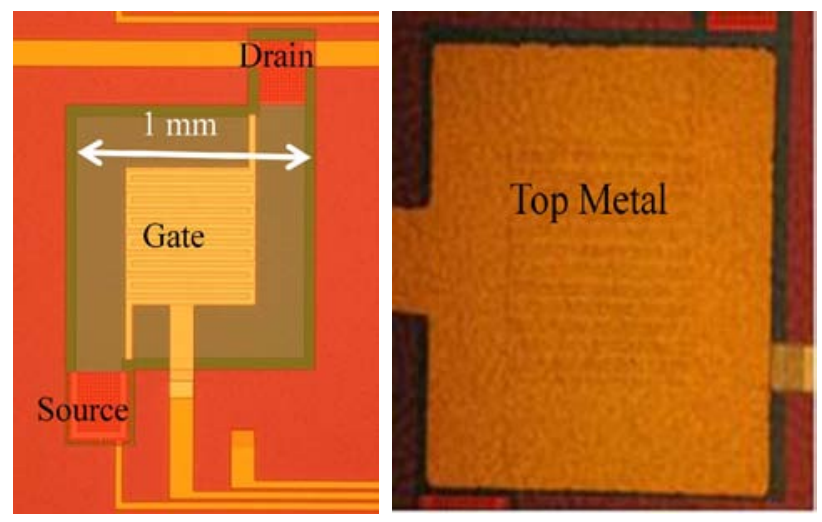

Fig. 2: Enlarged picture of fabricated POSFET devices before (left) and after (right) deposition and processing of PVDFTrFE piezoelectric polymer film. 

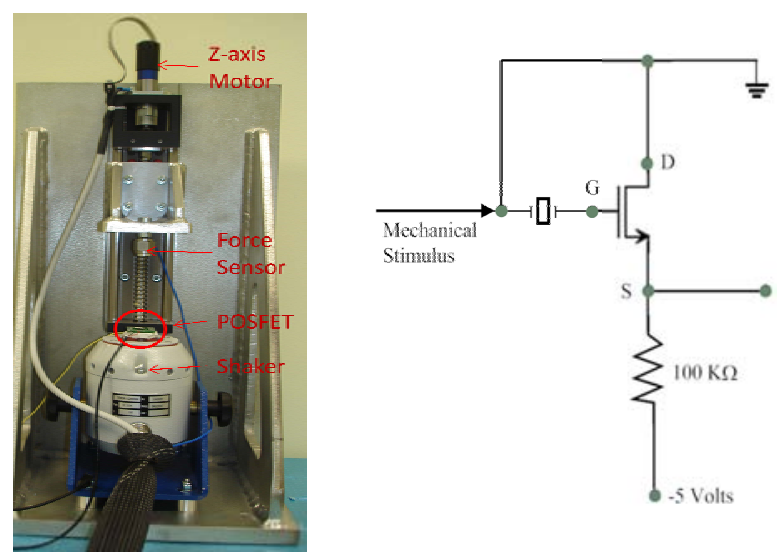

Fig. 3: (Left) Experiment set up. (Right) Connection scheme of POSFET devices used during testing.

steps of 50 volts each. Following these steps, the input and output characteristic of POSFET device are observed to be similar, both, before and after the polarization step [14].

\section{EXPERIMENT EVALUATION}

\section{A. Experiment Set Up}

The experiment set up used for testing POSFET touch sensing devices, is shown in Fig. 3. The TIRA shaker (Model - TV50018) on the set up can apply dynamic forces up to 18 $\mathrm{N}$ with frequency in the range $2 \mathrm{~Hz}-18 \mathrm{KHz}$. The force generated by the shaker is recorded by piezoelectric load cell (PCB Piezotronics, Model - 208C01), that can move along the z-axis. The POSFET touch sensor is placed on the shaker, as shown in Fig. 3 and then the load cell is brought in contact with it with help of the z-axis positioning motor. The POSFET device is thus pressed by vibration generator or shaker against the force sensor. To apply force on a touch sensor, special probes having diameter of $1 \mathrm{~mm}$ were developed. To ensure that the whole sensor is pressed, the diameter of the probe is slightly higher than the dimension of POSFET. These probes are attached to the load cell. The shaker, piezoelectric load cell and the z-axis positioning motor and the output of taxel are all measured and controlled through NI-DAQ device (USB-6259) which can acquire and control the data from 16 analog outputs, 24 digital I/O and 2 analog inputs.

\section{B. Experimental Results}

The response of POSFET touch sensing device, to dynamic normal forces, is recorded by connecting it in a sourcefollower circuit arrangement with floating gate, as shown in Fig. 3. Source-follower configuration results in less than unity gain, and hence POSFET devices can be tested for wide range of forces. Alternately, common source configuration can be used to have better force resolution.

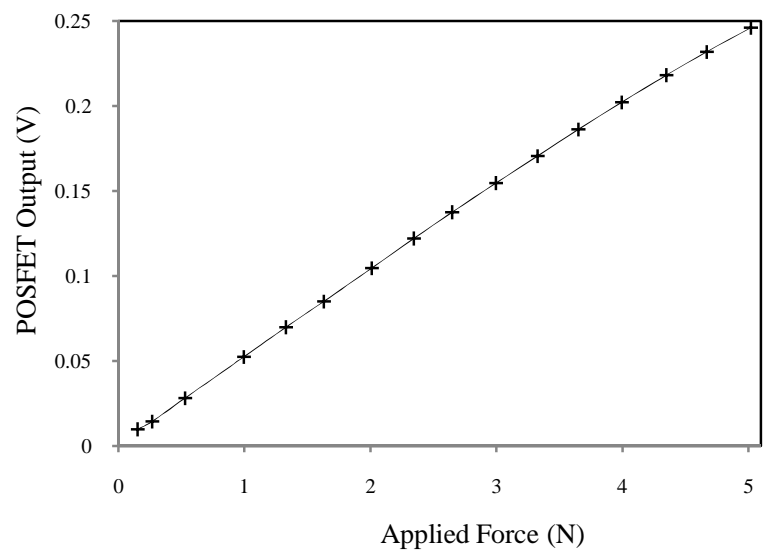

Fig. 4: Output of POSFET device versus $20 \mathrm{~Hz}$ force applied on top of the device, in normal direction.
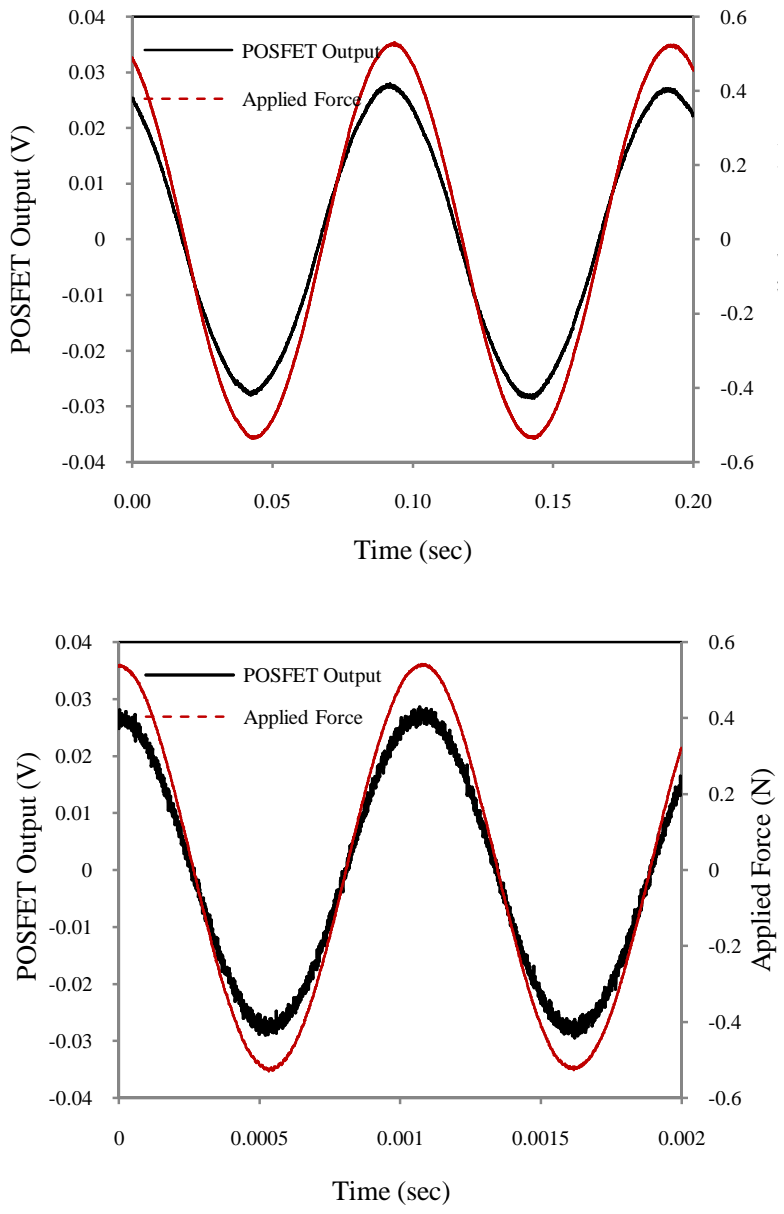

Fig. 5 POSFET response to $10 \mathrm{~Hz}$ and $925 \mathrm{~Hz}$ sine force.

The POSFET touch sensing device was tested for a range of dynamic normal forces $(0.15-5 \mathrm{~N}$, sinusoidal, $20 \mathrm{~Hz})$ and the output is shown in Fig. 4. The output of POSFET device is 


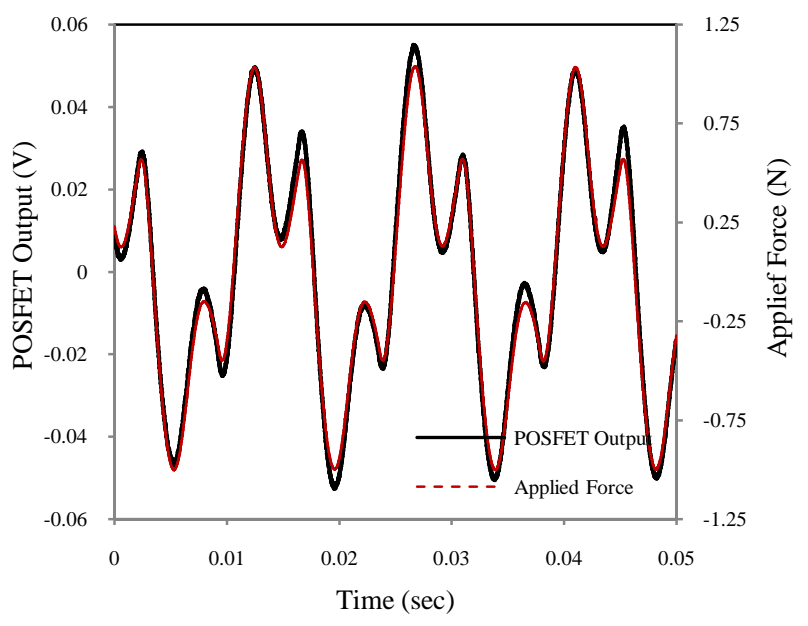

Fig. 6: POSFET response to a $70 \mathrm{~Hz}$ random periodic force.

linear, over the tested range, with a slope $49 \mathrm{mV} / \mathrm{N}$. This range of forces very well covers the range of forces, given in Table I, experienced by humans in normal manipulative tasks. At this stage, the applied force is restricted to $5 \mathrm{~N}$ to avoid risk of damaging the POSFET device. The output of POSFET touch sensing device, when a $10 \mathrm{~Hz}$ sinusoidal force is applied on top of the device, in normal direction, is shown in Fig. 5. The response of POSFET to a $925 \mathrm{~Hz}$ sinusoidal force is also shown in Fig. 5. The little noise in POSFET's output at 925 $\mathrm{Hz}$ is due to excessive mechanical vibration. The ratio of POSFET output and the corresponding applied force is same at both frequencies. Thus, POSFET touch sensing device can be used over a frequency range higher than that perceived by humans (see, Table I). Response of POSFET touch sensing device to a random periodic input force $(70 \mathrm{~Hz})$ is shown in Fig. 6. It can be noticed that POSFET devices are able to trace the applied force very well. The sensor was covered with a $300 \mu m$ thin protective PDMS (Polydimethylsiloxane) rubber, in all these experiments.

\section{CONCLUSION}

POSFET touch sensing devices, presented in this work, are able to match various features of human sense of touch. They show a linear response over range of forces $(0.15-5 \mathrm{~N})$ which is much wider than the forces experienced by humans in normal manipulative tasks. In addition to sensing and processing at same site and the improved performance, POSFET devices as an integral "sensotronic" unit offer practical advantages like reduction in number of wires - which is a key issue in robotics. The performance, utility and local processing capability of POSFET touch sensors can be further improved by including complex circuitry and following a SOC/SiP approach.
Though primarily designed for robotic applications, the POSFET touch sensing devices are also be suitable for medical applications because of the fact that impedance of PVDF-TrFE matches well with human tissues. POSFET touch sensing devices can also be used to obtain a conformal electronic surface by embedding them, as mechanically distinct and stiff sub circuit islands, in a soft and compliant polymer like PDMS and interconnect the islands with flexible and stretchable metallization. Alternately, they can also be developed on flexible electronic devices [18].

\section{ACKNOWLEDGEMENT}

This work is supported in part by the European Commission Project RobotCub (IST-FP6-004370) and in part by the Italian Ministry of Education University and Research under the project PRIN 2007 "Tactile Sensing System for Humanoid Robots using Piezo-polymer-FET devices".

\section{REFERENCES}

[1] R. H. LaMotte and M. A. Srinivasan, "Tactile Discrimination of Shape: Responses of Slowly Adapting Mechanoreceptive Afferents to a Step Stroked Across the Monkey Fingerpad," The Journal of Neuroscience, vol. 7, pp. 1655-1671, 1987.

[2] J. M. Wolfe, K. R. Kluender, D. M. Levi, L. M. Bartoshuk, R. S. Herz, R. L. Klatzky, and S. J. Lederman, Sensation and Perception. Sunderland, Massachusetts USA: Sinauer Associates Inc., 2006.

[3] R. S. Johannson and I. Birznieks, "First Spikes in Ensembles of Human Tactile Afferents Code Complex Spatial Fingertip Events," Nature Neuroscience, vol. 7, pp. 170-177, 2008.

[4] M. R. Cutkosky, R. D. Howe, and W. Provancher, "Force and Tactile Sensors," in Springer Handbook of Robotics. Berlin Heidelberg: Springer 2008, pp. 455-476.

[5] H. Takao, K. Sawada, and M. Ishida, "Monolithic Silicon Smart Tactile Image Sensor With Integrated Strain Sensor Array on Pneumatically Swollen Single-Diaphragm Structure," IEEE Tranactions on Electron Devices, vol. 53, pp. 1250-1259, 2006.

[6] B. J. Kane, M. R. Cutkosky, and G. T. A. Kovacs, "A Traction Stress Sensor Array for Use in High-Resolution Robotic Tactile Imaging" Journal of Microelectromechal Systems, vol. 9, 425434, 2000.

[7] T. Someya, T. Sekitani, S. Iba, Y. Kato, H. Kawaguchi, and T. Sakurai, " A Large-Area, Flexible Pressure Sensor Matrix with Organic Field-Effect Transistors for Artificial Skin Applications," Proceedings of National Academy of Sciences (PNAS), vol. 101, pp. 9966-9970, 2004.

[8] I. Manunza, A. Sulis, and A. Bonfiglio, "Pressure sensing by flexible, organic field effect transistors," Applied Physics Letters, vol. 89, p. 143502, 2006.

[9] D.-H. Kim, J.-H. Ahn, H.-S. Kim, K. J. Lee, T.-H. Kim, C.-J. Yu, R. G. Nuzzo, and J. A. Rogers, "Complementary Logic Gates and Ring Oscillators on Plastic Substrates by Use of Printed Ribbons of Single-Crystalline Silicon," IEEE Electron Devices Letters, vol. 29, pp. 73-76, 2008.

[10] R. S. Dahiya, M. Valle, and L. Lorenzelli, "SPICE Model of Lossy Piezoelectric Polymers," IEEE Transactions on Ultrasonics, Ferroelectrics and Frequency Control, vol. 56, pp. 387-396, 2009 
[11] R. G. Swartz and J. D. Plummer, "Integrated silicon-PVF2 acoustic transducer arrays," IEEE transactions on Electron Devices, vol. 26, pp. 1920-32, 1979.

[12] R. S. Dahiya, G. Metta, and M. Valle, "Development of Fingertip Tactile Sensing Chips for Humanoid Robots," in 5th IEEE International Conference on Mechatronics, Spain, 2009, pp 1-6.

[13] R. S. Dahiya, M. Valle, G. Metta, and L. Lorenzelli, "POSFET Based Tactile Sensor Arrays," in IEEE ICECS'07, The 14th International Conference on Electronics, Circuits and Systems, 2007, pp. 1075-1078.

[14] R. S. Dahiya, M. Valle, G. Metta, L. Lorenzelli, and A. Adami, "Design and Fabrication of POSFET Devices for Tactile Sensing " in TRANSDUCERS 2009, The 15th IEEE Int. Conf. on Solid-State Sensors, Actuators and Microsystems, Denver, USA, 2009 (in press).
[15] S. Martinoia, N. Rosso, M. Grattarola, L. Lorenzelli, B. Margesin, and M. Zen, "Development of ISFET array-based Microsystems for bioelectrochemical measurements of cell populations " Biosensors \& Bioelectronics, vol. 16, 1043-1050, 2001.

[16] R. S. Dahiya, M. Valle, L. Lorenzelli, G. Metta, and S. Pedrotti, "Deposition Processing and Characterization of PVDF-TrFE Thin Films for Sensing Applications," in IEEE Sensors 2008, Lecce, Italy., 2008, pp. 490-493.

[17] H. S. Nalwa, "Ferroelectric Polymers - Chemistry, Physics and Applications," New York: Marcel Dekker Inc., 1995.

[18] D.-H. Kim, J.-H. Ahn, W. M. Choi, H.-S. Kim, T.-H. Kim, J. Song, Y. Y. Huang, Z. Liu, C. Lu, and J. A. Rogers, "Stretchable and Foldable Silicon Integrated Circuits," Science, vol. 320, pp. 507-511, 2008. 